\title{
Three-Photon Luminescence of Gold Nanorods and Its Applications for High Contrast Tissue and Deep In Vivo Brain Imaging
}

\author{
Shaowei Wang ${ }^{1}$, Wang $\mathrm{Xi}^{2}$, Fuhong $\mathrm{Cai}^{1}$, Xinyuan $\mathrm{Zhao}^{3}$, Zhengping $\mathrm{Xu}^{3}$, Jun Qian ${ }^{1 凶}$, Sailing $\mathrm{He}^{1 凶}$ \\ 1. State Key Laboratory of Modern Optical Instrumentations, Centre for Optical and Electromagnetic Research, Zhejiang Provincial Key Laboratory \\ for Sensing Technologies, JORCEP (Sino-Swedish Joint Research Center of Photonics), Zhejiang University, Hangzhou, Zhejiang, 310058, China; \\ 2. Department of Neurobiology, Key Laboratory of Medical Neurobiology of Ministry of Health of China, Zhejiang Province Key Laboratory of \\ Neurobiology, School of Medicine, Zhejiang University, Hangzhou, Zhejiang, 310058, China; \\ 3. Bioelectromagnetics Laboratory, School of Medicine, Zhejiang University, Hangzhou, Zhejiang, 310058, China.
}

$\bowtie$ Corresponding authors: qianjun@zju.edu.cn and sailing@kth.se.

(c) Ivyspring International Publisher. This is an open-access article distributed under the terms of the Creative Commons License (http://creativecommons.org/ licenses/by-nc-nd/3.0/). Reproduction is permitted for personal, noncommercial use, provided that the article is in whole, unmodified, and properly cited.

Received: 2014.08.23; Accepted: 2014.11.05; Published: 2015.01.01

\begin{abstract}
Gold nanoparticles can be used as contrast agents for bio-imaging applications. Here we studied multi-photon luminescence (MPL) of gold nanorods (GNRs), under the excitation of femtosecond (fs) lasers. GNRs functionalized with polyethylene glycol (PEG) molecules have high chemical and optical stability, and can be used as multi-photon luminescent nanoprobes for deep in vivo imaging of live animals. We have found that the depth of in vivo imaging is dependent upon the transmission and focal capability of the excitation light interacting with the GNRs. Our study focused on the comparison of MPL from GNRs with two different aspect ratios, as well as their ex vivo and in vivo imaging effects under $760 \mathrm{~nm}$ and $1000 \mathrm{~nm}$ excitation, respectively. Both of these wavelengths were located at an optically transparent window of biological tissue (700-1000 nm). PEGylated GNRs, which were intravenously injected into mice via the tail vein and accumulated in major organs and tumor tissue, showed high image contrast due to distinct three-photon luminescence $(3 \mathrm{PL})$ signals upon irradiation of a $1000 \mathrm{~nm}$ fs laser. Concerning in vivo mouse brain imaging, the 3PL imaging depth of GNRs under $1000 \mathrm{~nm}$ fs excitation could reach $600 \mu \mathrm{m}$, which was approximately $170 \mu \mathrm{m}$ deeper than the two-photon luminescence (2PL) imaging depth of GNRs with a fs excitation of $760 \mathrm{~nm}$.
\end{abstract}

Key words: gold nanorods, three-photon luminescence, deep in vivo imaging, high contrast, tumor tissue.

\section{Introduction}

With the fusion of nanotechnology, biotechnology, and optical technology, various types of nanomaterials (e.g. carbon nanotubes [1], magnetic nanoparticles [2], and gold nanoparticles [3]) with unique optical properties have been applied in bio-imaging, bio-sensing and biotherapy. In particular, gold nanorods (GNRs) with well-defined shapes and sizes are very attractive, due to their ease of preparation and surface functionalization, low cytotoxicity, fine bio- compatibility, and excellent surface plasmon resonance (SPR) properties in the near-infrared (NIR) region $[4,5]$. All these features make GNRs very promising in biological applications, such as biomedical imaging and sensing, gene delivery, drug delivery and therapy $[5,6]$.

GNRs have two SPR bands: a strong longitudinal band which can be tuned by controlling the aspect ratio (length/diameter) of the GNRs, and a weak 
transverse band, which is located around $520 \mathrm{~nm}$ [4]. The peak wavelength and intensity of the longitudinal SPR band of GNRs are very sensitive to the local refractive index, and can be used as excellent biosensors to detect molecules which are bonded with or close to the surface of the GNRs [7, 8]. Their SPR property also makes GNRs very useful in surface-enhanced Raman spectroscopy (SERS) $[9,10]$. In addition, due to their strong plasmon resonant absorption band, which is tunable from the visible range to NIR, as well as their enhanced scattering cross section, GNRs have been well-developed for in vitro and in vivo bio-imaging, including plasmon-enhanced near-infrared imaging [9,11], dark-field scattering imaging [12], optical coherence tomography (OCT) [13], photoacoustic tomography (PAT) [14,15], two-photon luminescence imaging [16-18], etc.

During the past two decades, multi-photon luminescence microscopy has been developed and well suited for cell and tissue imaging. The upconverted MPL signals can be spectrally separated from the downconverted autofluorescence of biological samples very easily [19-22]. A multi-photon absorption/luminescence process has a nonlinear dependence on excitation intensity. This can help improve the spatial resolution of imaging and reduce the photobleaching of the sample outside the beam focus, as only the site where the laser beam is focused can be efficiently excited [21]. In addition, deep in vivo imaging is promising if the MPL excitation wavelength is selected in the spectral region of $650-1000 \mathrm{~nm}$, which is considered as an "optical tissue window", since light with a wavelength in this window has minimal optical absorption and relatively small scattering in biological tissue. GNRs exhibit strong plasmon-enhanced multi-photon luminescence (MPL, three or two-photon luminescence), and its brightness is much stronger than that of many existing conventional organic chromophores [16]. So far, many studies have demonstrated the potential of GNRs in cellular and in vivo MPL imaging [16-18]. Most of these studies focused on the two-photon luminescence (2PL) of GNRs, excited by a femtosecond ( $\mathrm{fs}$ ) laser with a wavelength between $700-800 \mathrm{~nm}$. The longitudinal band of the GNRs is also tuned to this spectral region to overlap with the MPL excitation wavelength. Hence, the 2PL efficiency of GNRs could be increased and the 2PL imaging depth could also be improved accordingly. However, in order to look deeper inside tissue, longer-wavelength fs excitation can be used [23]. Absorption loss of light increases when the excitation wavelength gets longer due to the absorption property of water, but scattering loss of light is reduced when longer-wavelength excitation is adopted, and thus the total light attenuation can be effectively reduced. Recently, several studies have demonstrated that longer-wavelength excited multi-photon luminescence can extend the maximum depth of in vivo imaging [23, 24]. In addition, under longer-wavelength (e.g. $>800 \mathrm{~nm})$ fs excitation, three-photon luminescence (3PL) of GNRs can be excited, and its luminescence spectrum is located between one-third of the excitation wavelength and half of the excitation wavelength. A high-order nonlinear optical signal is helpful in improving the imaging contrast [25]. To achieve deeper and higher-contrast bio-imaging, the longitudinal band of the GNRs can be tuned to overlap with the MPL excitation wavelength in the longer-wavelength region of the optical tissue window (e.g. $1000 \mathrm{~nm})$.

In this study, we demonstrated that PEGlylated GNRs can be employed as 3PL luminescent nanoprobes for high contrast tissue and deep in vivo mouse brain imaging. A conventional and an improved seed-mediated growth method were used to prepare GNRs with longitudinal LSPR peaks at $760 \mathrm{~nm}$ and $1000 \mathrm{~nm}$ (abbreviated as 760GNRs and 1000GNRs), respectively. The GNRs showed excellent MPL (2PL and 3PL) signals upon fs excitation at $760 \mathrm{~nm}$ and $1000 \mathrm{~nm}$, and MPL in vitro and in vivo imaging experiments were performed by treating mice with PEGylated GNRs. 3PL of 1000GNRs, which accumulated in major organs and tumor tissue, showed strong contrast signals under $1000 \mathrm{~nm}$-fs excitation. In addition, the in vivo 3PL brain imaging depth of 1000GNRs under $1000 \mathrm{~nm}$-fs excitation could reach $600 \mu \mathrm{m}$, approximately $170 \mu \mathrm{m}$ deeper than the 2PL imaging depth of 760GNRs with fs-excitation at 760 $\mathrm{nm}$. Compared to 2PL imaging of 760GNRs excited at $760 \mathrm{~nm}$ fs, 3PL imaging of 1000GNRs excited at 1000 $\mathrm{nm}$ fs possesses several advantages, including weak background autofluorescence, high signal to noise ratio, low photobleaching and deep tissue penetration without causing photodamage.

\section{Materials and Methods}

\section{Materials}

All chemicals were obtained from commercial suppliers and used without further purification. Cetyltrimethylammonium bromide (CTAB $>98.0 \%$ ), hydrogen tetrachloroaurate (III) trihydrate $\left(\mathrm{HAuCl}_{4} \bullet 3 \mathrm{H}_{2} \mathrm{O}\right)$, sodium borohydride $\left(\mathrm{NaBH}_{4}, 99 \%\right)$, silver nitrate $\left(\mathrm{AgNO}_{3},>99 \%\right)$, L-ascorbic Acid (BioUltra, 299.5\%) and hydrochloric acid $(\mathrm{HCl}, 37$ wt.\% in water) were purchased from Sigma-Aldrich. Sodium oleate $(\mathrm{NaOL},>97.0 \%$ ) was purchased from TCI America. SH-PEG- $\mathrm{CH}_{3}$ (methoxy PEG Thiol, MW5000) was purchased from JenKem Technology Co., Ltd. Deionized (DI) water was used in all ex- 
periments.

\section{Synthesis of seeds for gold nanorods (GNRs)}

GNRs with longitudinal LSPR peaks located at 650-800 nm (short GNRs) and 840-1100 nm (long GNRs) were synthesized using two protocols based on the seed-mediated method in an aqueous solution proposed by Nikoobakht [26] and Xingchen Ye [27], respectively. The synthesis of seeds for both methods is the same. $0.6 \mathrm{ml}$ of $0.01 \mathrm{M}$ ice-cold sodium borohydride was quickly injected into a $10 \mathrm{ml}$ stirring (1200 rpm) aqueous solution containing $0.25 \mathrm{mM} \mathrm{HAuCl}_{4}$ and $0.1 \mathrm{M} \mathrm{CTAB}$. The solution color changed from yellow to brownish yellow. After stirring the solution vigorously for $2 \mathrm{~min}$, the solution was kept for $30 \mathrm{~min}$ at room temperature in order to allow the hydrolysis of $\mathrm{NaBH}_{4}$ before use.

\section{Growth of short GNRs}

The short GNR growth solution was prepared by adding $0.2 \mathrm{ml}$ of $25 \mathrm{mM} \mathrm{HAuCl}_{4}$ to $5 \mathrm{ml}$ of $0.2 \mathrm{M}$ CTAB. Then, $16 \mathrm{mM}$ of silver nitrate $\left(\mathrm{AgNO}_{3}\right)$ and 80 $\mathrm{mM}$ of ascorbic acid were separately added to this solution to make a final mixture volume of about 10 $\mathrm{ml}$. After the growth solution turned colorless, $12 \mu \mathrm{l}$ of previously prepared seed solution was injected into it. Then it was left undisturbed at $37^{\circ} \mathrm{C}$ for $18 \mathrm{~h}$ to let the GNRs grow. The GNR solution was centrifuged twice at $7200 \mathrm{rpm}$ for $10 \mathrm{~min}$ to remove the excess reaction reagents and then redispersed into DI water. GNRs with different longitudinal plasmon bands could be acquired through controlling the aspect ratios by tuning the additions of $\mathrm{AgNO}_{3}$ and ascorbic acid. To make GNRs with the longitudinal LSPR peaks at 650 $\mathrm{nm}$ and $760 \mathrm{~nm}$, the amount of $\mathrm{AgNO}_{3}$ was tuned to $40 \mu \mathrm{l}$ and $90 \mu \mathrm{l}$ and ascorbic acid was tuned to $60 \mu \mathrm{l}$ and $75 \mu \mathrm{l}$, respectively.

\section{Growth of long GNRs}

A long GNR growth solution containing $3.5 \mathrm{~g}$ CTAB and $0.617 \mathrm{~g} \mathrm{NaOL}$ was prepared in warm water $\left(\sim 30^{\circ} \mathrm{C}\right)$ in a $500 \mathrm{ml}$ Erlenmeyer flask. $12 \mathrm{ml}$ of $4 \mathrm{mM}$ $\mathrm{AgNO}_{3}$ solution and $125 \mathrm{ml}$ of $1 \mathrm{mM} \mathrm{HAuCl}_{4}$ were added. After $60 \mathrm{~min}$ of stirring, when the mixture solution became colorless, $2.4 \mathrm{ml}$ of $\mathrm{HCl}$ (37 wt. \% in water) and $0.625 \mathrm{ml}$ of $64 \mathrm{mM}$ ascorbic acid were added, and the solution was vigorously stirred for 1 min. After that, $0.4 \mathrm{ml}$ of seed solution was injected into the growth solution, which was stirred for $30 \mathrm{~s}$ and left undisturbed at $37^{\circ} \mathrm{C}$ for $12 \mathrm{~h}$ to let the GNRs grow. The GNR solution was centrifuged twice at $6000 \mathrm{rpm}$ for $10 \mathrm{~min}$ to remove the excess reaction reagents and then redispersed into DI water. The obtained GNRs have a longitudinal LSPR peak at about $1000 \mathrm{~nm}$. For other peaks see details in Additional file 1: Table S1.

\section{Surface modification of GNRs}

For typical PEG modification of GNRs, $10 \mathrm{ml}$ of the as-prepared GNRs were centrifuged twice at 6000 rpm (7200 rpm for short GNRs) for $10 \mathrm{~min}$, and then the precipitate was dispersed in $5 \mathrm{ml}$ of a $2 \mathrm{mg} / \mathrm{ml}$ aqueous solution of $\mathrm{SH}-\mathrm{PEG}-\mathrm{CH}_{3}(\mathrm{MW}=5000)$. The solution was immediately vortexed for $3 \mathrm{~min}$ for uniform mixing and stirred magnetically for $12 \mathrm{~h}$. After that, the solution was centrifuged and the precipitate was redispersed in $5 \mathrm{ml}$ of a $2 \mathrm{mg} / \mathrm{ml}$ PEG aqueous solution to perform the same procedure, making sure that the $\mathrm{CTAB} / \mathrm{NaOL}$ molecules were completely displaced by PEG molecules. Successful surface modification with PEG was assessed using UV-vis-NIR spectroscopy and Zeta potentials (Additional file 1: Figure S1, S2). The final PEGylated GNR solution was washed twice and dispersed in a $1 \times$ PBS solution for in vivo use.

\section{Characterizations of GNRs}

The absorption spectra of GNRs were measured by a Shimadzu UV3600 UV-vis-NIR scanning spectrophotometer. TEM images were captured by a JEOL JEM-1200EX microscope operated at $160 \mathrm{kV}$. TEM samples were prepared by casting $10 \mu \mathrm{l}$ of GNR aqueous solution on copper grids and dried at room temperature. Zeta potentials were measured on a Zetasizer 3000 HAS instrument. The multi-photon luminescence spectra of GNRs excited by the $1000 \mathrm{~nm}$ and $760 \mathrm{~nm}$ fs laser were measured by a home-built cage system and collected by an optical fiber spectrometer (PG2000, Ideaoptics Instruments).

\section{Cell viability analysis}

The cytotoxicity of GNRs to HeLa cells was evaluated by following the instructions of cell counting kit-8 (CCK-8). 5000 cells/well in a $100 \mu \mathrm{l}$ suspension were incubated in 96-well plates for $24 \mathrm{~h}$. Then, a $100 \mu \mathrm{l}$ fresh culture medium containing GNRs with various concentrations ranging from 0 to $200 \mathrm{pM}$ was added into each well. After incubation for $24 \mathrm{~h}$, the culture medium was removed and the cell well was washed three times with PBS. In the end, $100 \mu \mathrm{l}$ culture medium containing CCK-8 (10\%) was added into each well for $2 \mathrm{~h}$, and the absorbance was measured at $450 \mathrm{~nm}$ by using a microplate reader (Thermo, USA).

\section{Animals' preparation}

All the animal experiments were performed strictly in compliance with the requirements and guidelines of the Institutional Ethical Committee of Animal Experimentation of Zhejiang University. The BALB/c mice $(\sim 20 \mathrm{~g}$, male) and nude mice $(\sim 18 \mathrm{~g}$, male) were obtained from the Laboratory Animal Center of Zhejiang University (Hangzhou, China). 
The mice were housed in cages at $24{ }^{\circ} \mathrm{C}$ with a $12 \mathrm{~h}$ light/dark cycle and were fed with standard laboratory water and chow. Tumor-bearing mice were prepared as follows. HeLa cells were cultured in the Dulbecco's minimum essential media (DMEM) containing 10\% fetal bovine serum (FBS), 1\% penicillin, and $1 \%$ amphotericin $\mathrm{B}$ under an atmosphere environment of $5 \% \mathrm{CO}_{2}$ and $95 \%$ air at $37^{\circ} \mathrm{C}$. The incubated HeLa cells were injected subcutaneously into the abdomen of nude mice with a dose of $3 \times 10^{6}$ cells in $200 \mu \mathrm{l}$ of $1 \times$ PBS. Tumor growth was monitored every 2 days until a tumor size of approximately 5 $\mathrm{mm}$ in diameter was observed.

\section{Tissue slices preparation}

PEG-760GNRs and PEG-1000GNRs (in $200 \mu 11 \times$ PBS, $25 \mathrm{nM}$ ) were intravenously injected into normal mice and tumor-bearing mice via the tail vein, respectively, and the two GNR samples (in $50 \mu l 1 \times$ PBS, 5 $\mathrm{nM}$ ) were intradermally injected into the left forepaw pad of the mice. After the intravenous injections, GNRs can be transported to the major organs via blood circulation [28]. The intradermal injections make it easy for GNRs to be transported to the sentinel lymph node via the lymph vessels $[29,30]$. All the control mice (normal and tumor-bearing mice) were treated with an equal amount of saline. Major organs (liver, brain, \& kidney) and the sentinel lymph node (SLN) were excised at $0.5 \mathrm{~h}$ and tumors were extracted $48 \mathrm{~h}$ after the injection. All the tissue samples were fixed in $4 \%$ paraformaldehyde to preserve the tissue architecture, and then transferred to $70 \%$ ethanol prior to processing. Then they were embedded in paraffin, sectioned at $50 \mu \mathrm{m}$ thicknesses and mounted on slides. The slides were covered with coverslips and stored at $4{ }^{\circ} \mathrm{C}$ for imaging use.

\section{In vitro MPL imaging of GNRs in tissue}

The MPL images of GNRs accumulated in tissues were recorded by a commercial upright multi-photon scanning microscope (Olympus, FV1000). The fs laser beam was focused onto the sample by a 20 $\times$ objective $(\mathrm{NA}=0.75)$, and the MPL signals were collected by a photomultiplier tube through non-descanned detection.

\section{In vivo 3PL imaging of GNRs in mouse ear blood vessels}

$200 \mu 11 \times$ PBS solution of PEG-1000GNRs (25 $\mathrm{nM})$ were intravenously injected into male BALB/c mice. The mice in the control group were intravenously injected with $200 \mu \mathrm{l}$ saline. The mouse was anesthetized and placed on a Petri dish with one ear attached to the coverslip and prepared for in vivo imaging within 15 minutes [16]. An upright multi-photon scanning microscope was used to image the blood vessels. The $1000 \mathrm{~nm}$ fs laser beam was focused by a water-immersion objective lens $(25 \times$, NA $=1)$ onto the earlobe immersed by water, and the 3PL signals were collected by a photomultiplier tube through non-descanned detection with a filter within the $420-460 \mathrm{~nm}$ range. We acquired $80-\mu \mathrm{m}$-deep stacks for 3PL of PEG-1000GNRS taken with a $1 \mu \mathrm{m}$ step depth.

\section{In vivo MPL imaging of GNRs in mouse brain blood vessels}

A cranial window microsurgery on the mouse brain was performed. Briefly, the mouse was anesthetized and a small piece of skull was excised using a dental drill. The surgery was operated under sterile conditions to avoid infections and any damage to the dura mater, and to ensure that the mice could live well after the imaging experiments. For in vivo experiments, the mice were injected with GNRs, and then anesthetized and placed on a home-made setup within 15 minutes for imaging, with their brain immobilized. To compare $1000 \mathrm{~nm}$ fs laser and $760 \mathrm{~nm}$ fs laser excited MPL, $200 \mu 11 \times$ PBS mixed solution of PEG-760GNRs (100 $\mu \mathrm{l}, 25 \mathrm{nM})$ and PEG-1000GNRs $(100 \mu \mathrm{l}, 25 \mathrm{nM})$ were intravenously injected into the mice. The dosage of PEG-GNRs used in our in vivo studies is reasonable compared with other literatures $(200 \mu \mathrm{l} \mathrm{of} 50 \mathrm{mg} / \mathrm{ml}$ GNRs in [31] and $100 \mu \mathrm{l}$ of 10 $\mathrm{mg} / \mathrm{mL}$ in [32], our dosage is around $100 \mu \mathrm{l}$ of 6 $\mathrm{mg} / \mathrm{mL}$ ). Meanwhile this dosage is safe for in vivo studies as the biocompatibility of PEG-GRNs had been verified by the histopathology test (Additional file 1: Figure S3). In addition, mice injected with $200 \mu 1$ saline were also imaged as the control group. An upright multi-photon scanning microscope was used to image the blood vessels. For $1000 \mathrm{~nm}$ excitation, the fs laser beam was focused by a water-immersion objective lens $(25 \times)$ onto the brain surface, which was immersed in water. The 3PL signals were collected by a photomultiplier tube through non-descanned detection with a filter within the $420-460 \mathrm{~nm}$ range. For $760 \mathrm{~nm}$ excitation, the 2PL signals were collected by the PMT with two filters within 500-540 $\mathrm{nm}$ and 575-630 $\mathrm{nm}$ ranges.

\section{Histology}

Tissue samples were harvested and fixed in $4 \%$ paraformaldehyde overnight at $4{ }^{\circ} \mathrm{C}$. Then the samples were embedded in paraffin, sectioned, and stained with hematoxylin and eosin (H\&E).

\section{Results and Discussion}

\section{Synthesis and Characterizations of GNRs}

GNRs with low and high aspect ratios (corresponding to short and long GNRs) were synthesized 
using two different protocols based on seed-mediated methods in aqueous solution. The short GNRs were synthesized by a conventional method proposed by Nikoobakht [26], with the longitudinal LSPR peak tunable from $600 \mathrm{~nm}$ to $800 \mathrm{~nm}$. However, it is a little bit difficult to use this approach to synthesize GNRs with a longitudinal LSPR peak longer than $800 \mathrm{~nm}$. Thus, an improved technology using a binary surfactant mixture was adopted to tune the longitudinal LSPR peak of GNRs from $800 \mathrm{~nm}$ to $1100 \mathrm{~nm}$ [27]. Figure 1 showed the normalized absorption spectra (Figure 1a) and transmission electron microscopy (TEM) images (Figure 1b) of the GNRs with different aspect ratios. According to the conventional method, we prepared short GNRs with longitudinal LSPR peaks of $650 \mathrm{~nm}$ and $760 \mathrm{~nm}$ by tuning the amounts of $\mathrm{AgNO}_{3}$ and ascorbic acid (see Materials and Methods for details). By utilizing the improved method, we obtained long GNRs with longitudinal LSPR peaks of $840 \mathrm{~nm}, 900 \mathrm{~nm}, 1000 \mathrm{~nm}, 1036 \mathrm{~nm}, 1060 \mathrm{~nm}$ and 1100 $\mathrm{nm}$. The improved new method used the combination of $\mathrm{CTAB}$ and sodium oleate $(\mathrm{NaOL})$ as surfactants, in which the concentration of CTAB in the growth solution could be reduced to as low as $0.037 \mathrm{M}$. In the conventional method this concentration is $0.1 \mathrm{M}$. The improved method can achieve a narrow and uniform size distribution of GNRs. Furthermore, in the normalized absorption spectra (the peak intensities of longitudinal SPR bands of all the GNRs were normalized), the intensities of transverse SPR bands of long GNRs were much lower than those of short GNRs (as shown in Figure 1a), indicating that the improved synthesis method could also achieve a high yield of GNRs with very few byproducts (e.g. gold nanospheres). We chose two kinds of GNRs with longitudinal LSPR peaks at $760 \mathrm{~nm}$ (760GNRs) and $1000 \mathrm{~nm}$ (1000GNRs) for further imaging study. Representative TEM images showed that 760GNRs (Figure 1 d) were $\sim 20 \times 65 \mathrm{~nm}$ with aspect ratio (AR) $\sim 3.2$, and 1000GNRs (Figure 1f) were $\sim 15 \times 100 \mathrm{~nm}$, with aspect ratio $(\mathrm{AR}) \sim 6.5$. As shown in Figure $1 \mathrm{~b}$, the longitudinal LSPR peak wavelengths had a linear relationship with the aspect ratios of GNRs, as previously reported [33].
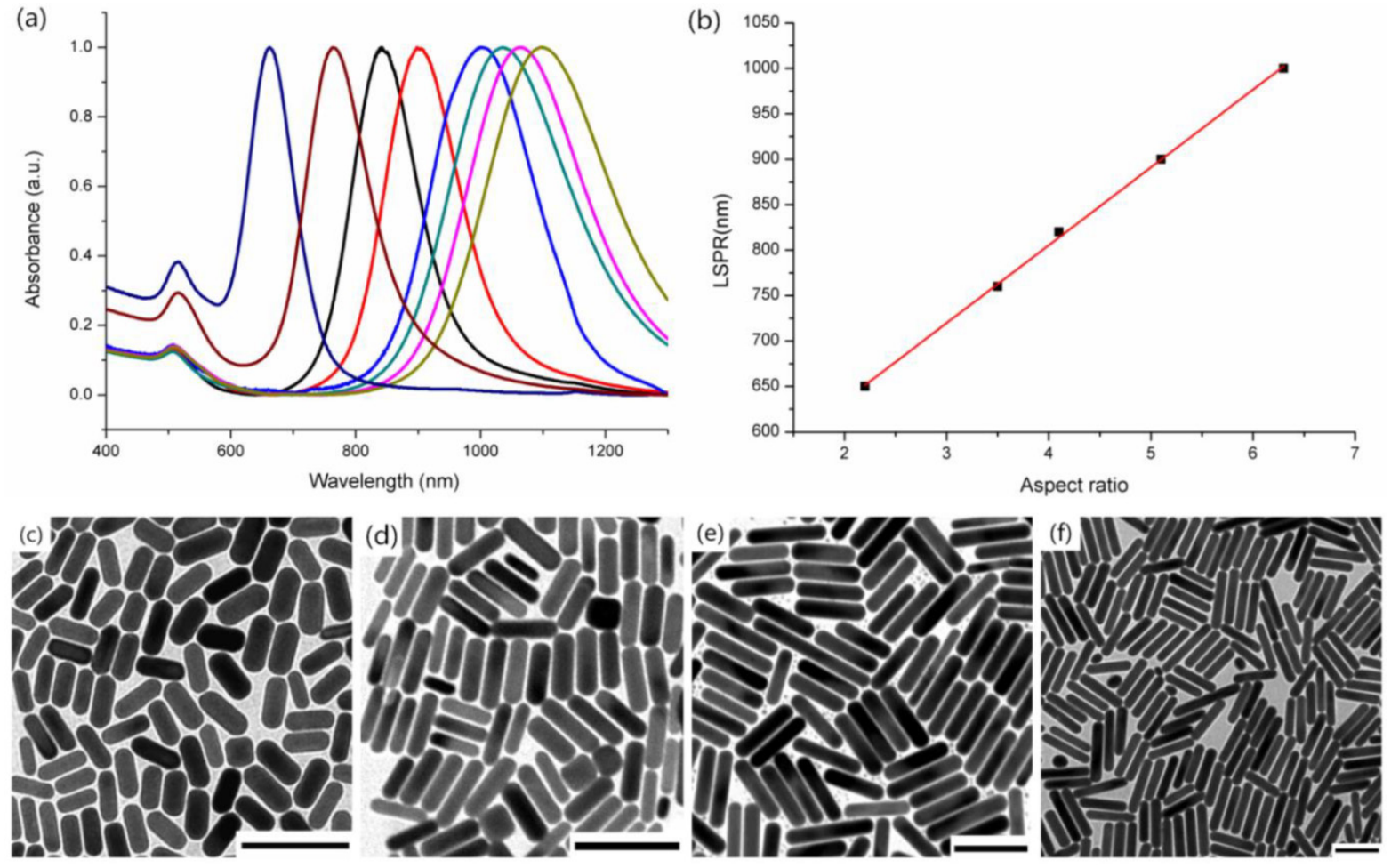

Figure 1. Characterizations of gold nanorods. (a) Representative UV-vis-NIR absorption spectra of GNRs with the longitudinal localized surface plasmon resonance (LSPR) peaks at $650 \mathrm{~nm}, 760 \mathrm{~nm}, 840 \mathrm{~nm}, 900 \mathrm{~nm}, 1000 \mathrm{~nm}, 1036 \mathrm{~nm}, 1060 \mathrm{~nm}$ and $1100 \mathrm{~nm}$ (from left to right). (b) The relationship between the longitudinal LSPR peaks and calculated aspect ratios (based on TEM images). The straight line is a linear fitting of data points. (c-f) Representative TEM images of GNRs with the longitudinal LSPR peaks at (c) $650 \mathrm{~nm}$, (d) $760 \mathrm{~nm}$, (e) $920 \mathrm{~nm}$, and (f) $1000 \mathrm{~nm}$. Scale bars: $100 \mathrm{~nm}$. 


\section{Surface modification of GNRs for bioimaging}

The as-prepared GNRs were positively charged due to the presence of CTAB for short GNRs and a binary surfactant mixture composed of $C T A B$ and NaOL for long GNRs. To graft PEG molecules on the surface of GNRs, a reaction was formed with CTAB/NaOL-coated GNRs and thiol-PEG under vigorous stirring in order to displace the $\mathrm{CTAB} / \mathrm{NaOL}$ layer with PEG polymer through the strong Au-S binding (see Materials and Methods for details). A PEG polymer is a kind of biocompatible polymer, and it can keep GNRs chemically stable, as well as improve the long circulation of GNRs in an animal body during an in vivo experiment [34]. After PEG modification, the absorption spectra of PEG-GNRs were recorded, which were very similar with that of CTAB/NaOL-GNRs, and had little red-shift of the longitudinal LSPR peaks. This illustrates a successful displacement of $\mathrm{CTAB} / \mathrm{NaOL}$ molecules by a PEG polymer on the surface of GNRs that was achieved without aggregation (Additional file 1: Figure S1). The Zeta-potential testing of the GNRs was performed to measure the surface charge of the GNRs, as well as to double check the coatings of the PEG molecules on the surface of the GNRs. Both CTAB-GNRs and CTAB/NaOL-GNRs showed positive charges, while for the PEGylated GNRs, the zeta-potential was nearly neutral (Additional file 1: Figure S2). The resulting GNRs were referred to as PEG-GNRs and utilized for the following studies.

\section{Stability and Cytotoxicity of GNRs}

Although the stability and cytotoxicity of PEG modified CTAB-GNRs have been studied by some previous works $[9,34]$, they have not been completely understood for PEG modified CTAB/NaOL-GNRs. Therefore, we first recorded the absorption spectra of PEG-GNRs in an aqueous solution with $\mathrm{pH}$ values varying from 1 to 13, as well as in an animal serum and PBS solution at the beginning. $48 \mathrm{~h}$ later, the absorption spectra of PEG-GNRs in different solutions were measured again, and various ratios could be obtained by dividing the intensities at longitudinal LSPR peaks of PEG-GNRs with those at the beginning (Figure 2a). We found that the ratios in all solutions were larger than $90 \%$, indicating that the optical and chemical properties of PEG-GNRs were maintained very well in various extreme chemical and biological conditions.

The cytotoxicity of PEG-GNRs towards cells was also examined by following the instructions of cell counting kit-8 (CCK-8). Figure $2 \mathrm{~b}$ showed the relative viabilities of HeLa cells treated with PEG-GNRs or CTAB/NaOL-GNRs after $24 \mathrm{~h}$. The cells treated with PEG-GNRs maintained very high viabilities, and it was still larger than $85 \%$ even when the concentration of PEG-GNRs was as high as 200 pM. However, CTAB/NaOL-GNRs exhibited high cytotoxicity $24 \mathrm{~h}$ after the cell treatment (only 25\%), due to the existence of CTAB molecules [33]. Furthermore, in vivo biocompatibility of PEG-GNRs was assessed by using a histopathology test (Additional file 1: Figure S3). The results show that at $24 \mathrm{~h}, 48 \mathrm{~h}$ and $72 \mathrm{~h}$ post-administration of PEGylated GNRs, mice have no inflammation or abnormalities on their major organs during the GNRs accumulation via the blood circulation, suggesting that GNRs are highly biocompatible. The low cytotoxicity and high biocompatibility of PEG-GNRs make them excellent optical probes for various in vitro and in vivo bio-imaging applications.
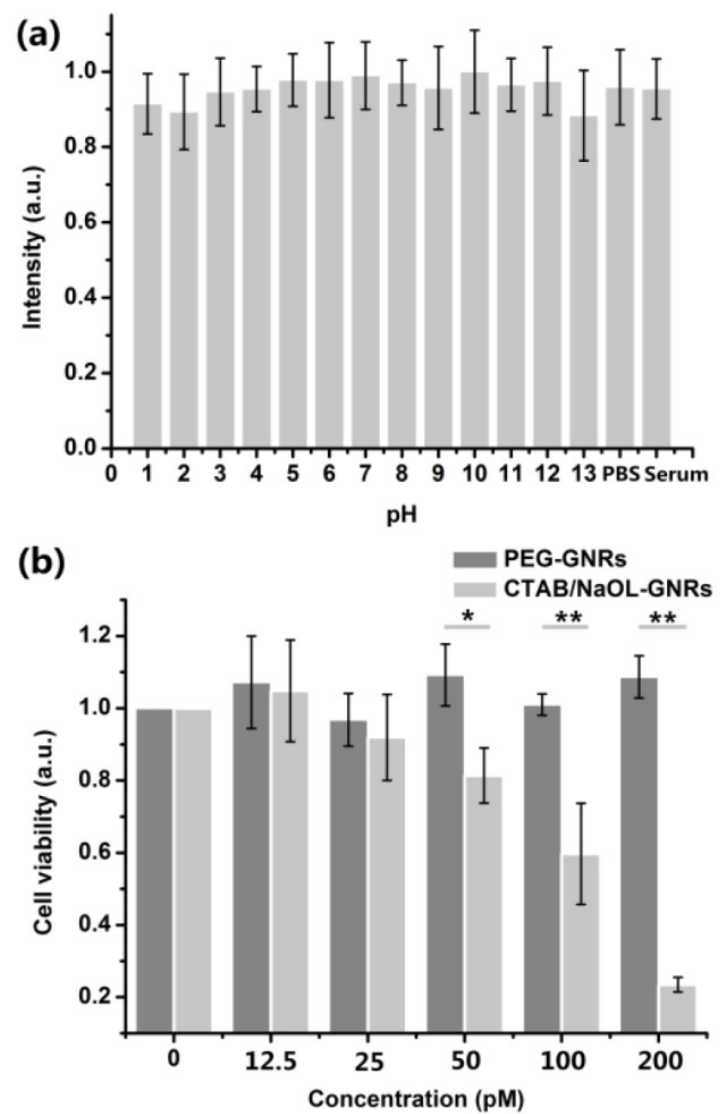

Figure 2. (a) Absorption intensity ratios of the longitudinal LSPR wavelength of PEGylated GNRs, measured immediately after and $48 \mathrm{~h}$ after dispersion in solutions with different $\mathrm{pH}$ values, serums and PBS solutions. (b) Viability of HeLa cells after incubation with the CTAB/NaOL GNRs and PEGylated GNRs

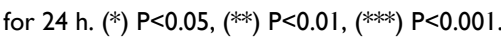

\section{MPL properties of GNRs}

Three/two photon luminescence processes of GNRs occur when three/two photons are absorbed by a GNR and electron-hole pairs are generated by the exciting electrons from d-band to sp-band. The excited electrons then move to the Fermi level by losing 
energy, and recombination of the electron-hole pairs results in 3PL/2PL emission [16, 35, 36]. Multiphoton luminescence (3PL and 2PL) from GNRs can be excited by femtosecond lasers that have ultrashort pulses with high peak intensities [23]. When the GNRs were excited with a fs laser, we observed a broad MPL spectrum in the visible region (Figure 3a). From the emission spectra we can see that the peaks are around 550-650 nm, which are expected to originate from the interband transitions near the $\mathrm{X}$ and $\mathrm{L}$ symmetry points of the first Brillouin zone of gold crystal $[35,36,37]$. The MPL spectra of GNRs excited by $1000 \mathrm{~nm}$ and $760 \mathrm{~nm}$ fs lasers were consistent with the results in some previously reported works $[16,35]$. For the 3PL of GNRs excited by a $1000 \mathrm{~nm}$ fs laser, the emission wavelength band locates between the $1 / 3$ and $1 / 2$ of the excitation wavelength, namely from around $330 \mathrm{~nm}$ to $500 \mathrm{~nm}$. For the 2PL of GNRs excited by $1000 \mathrm{~nm}$ and $760 \mathrm{~nm}$, their emission wavelengths are longer than $500 \mathrm{~nm}$ and $380 \mathrm{~nm}$, respectively $[25,38]$. The $3 \mathrm{PL}$ and $2 \mathrm{PL}$ were confirmed by examination of the dependence of luminescence intensity on the excitation power in three spectral regions $(420-460 \mathrm{~nm}, 500-540 \mathrm{~nm}$ and $575-630 \mathrm{~nm})$. The multi-photon luminescence from 1000GNRs excited by the $1000 \mathrm{~nm}$ fs laser consists of both three-photon
(420-460 nm, 3PL) and two-photon (500-540 nm and 575-630 nm, 2PL) emissions [25]. The emission intensity (integrated from 420 to $460 \mathrm{~nm}$ ) of 1000GNRs is linearly proportional to the cube of the $1000 \mathrm{~nm}-\mathrm{fs}$ laser excitation power (Figure $3 b$ ), and the emission intensity (integrated from 500 to $540 \mathrm{~nm}$ and from 575 to $630 \mathrm{~nm}$ ) is linearly proportional to the square of the $1000 \mathrm{~nm}$-fs laser excitation power (Figure 3c), confirming the three-photon and two-photon excitation processes. As for 760GNRs excited by the $760 \mathrm{~nm}$ fs laser, the luminescence was a two-photon excitation process (Additional file 1: Figure S4).

\section{High contrast 3PL imaging for evaluating dis- tribution of GNRs in tissues.}

GNRs have been widely used in the nanomedicine field for diagnostic and therapeutic purposes [6, $39,40]$. For example, they can serve as carriers for the delivery of drugs and genetic materials, and they can also be used as a medicinal or diagnostic agent for the treatment of tumors [5, 6, 39-44]. However, the biodistribution behavior of GNRs still needs to be carefully assessed before practical clinical applications. Herein, 3PL imaging was utilized to evaluate the accumulation and distribution of intravenously injected GNRs in tissues of normal and tumor-bearing mice. (a)

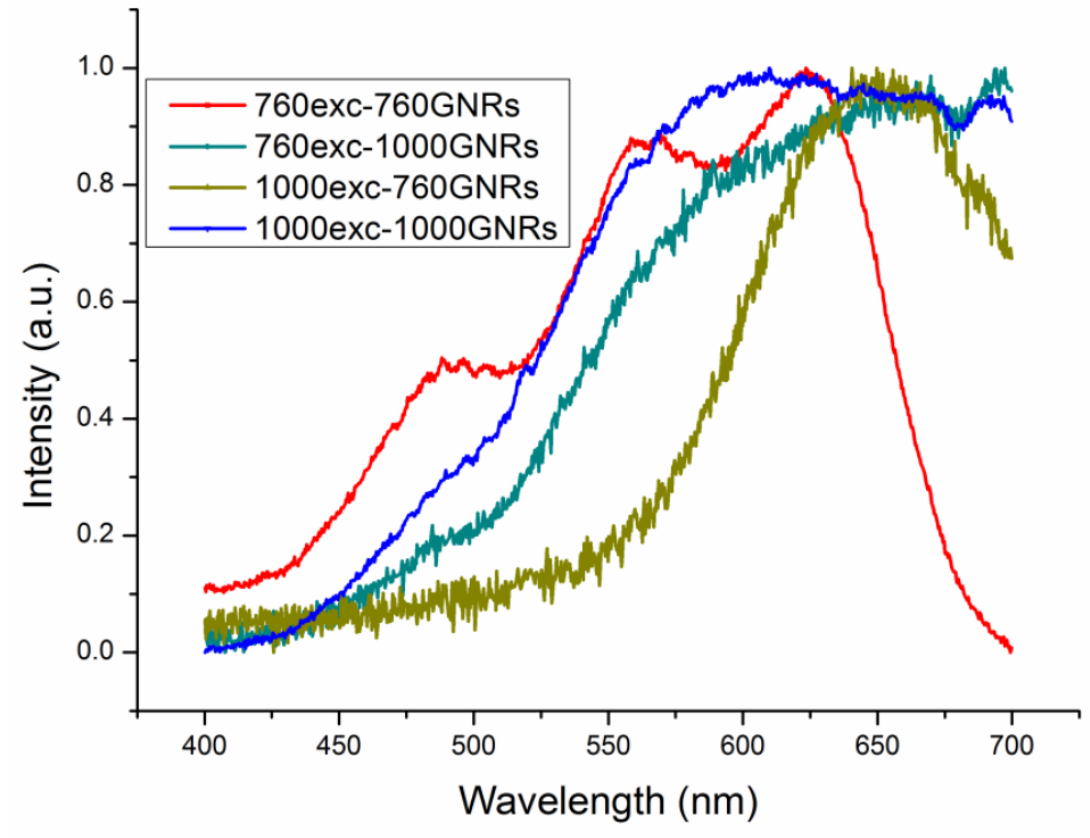

(b)

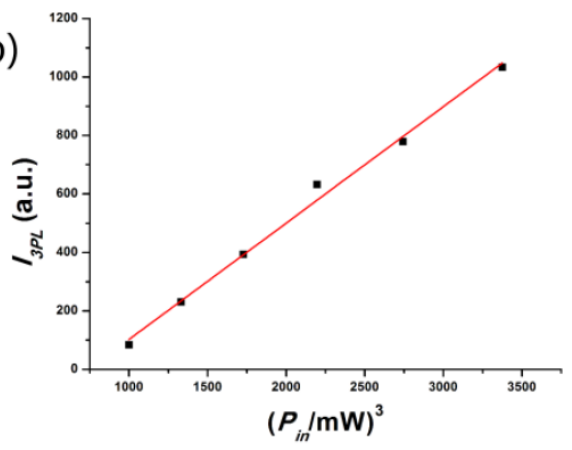

(c)

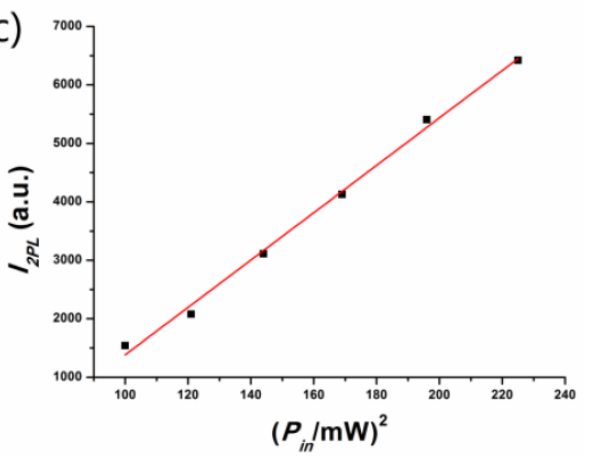

Figure 3. Three-photon luminescence (3PL) and two-photon luminescence (2PL) from GNRs. (a) Emission spectra from GNRs excited by a $1000 \mathrm{~nm}$ and $760 \mathrm{~nm}$ fs laser. (b) Cube dependence of 3PL from 1000GNRs on the excitation power of the $1000 \mathrm{~nm}$ fs laser. (c) Square dependence of $2 \mathrm{PL}$ from $1000 \mathrm{GNRs}$ on the excitation power of the $1000 \mathrm{~nm}$ laser. 

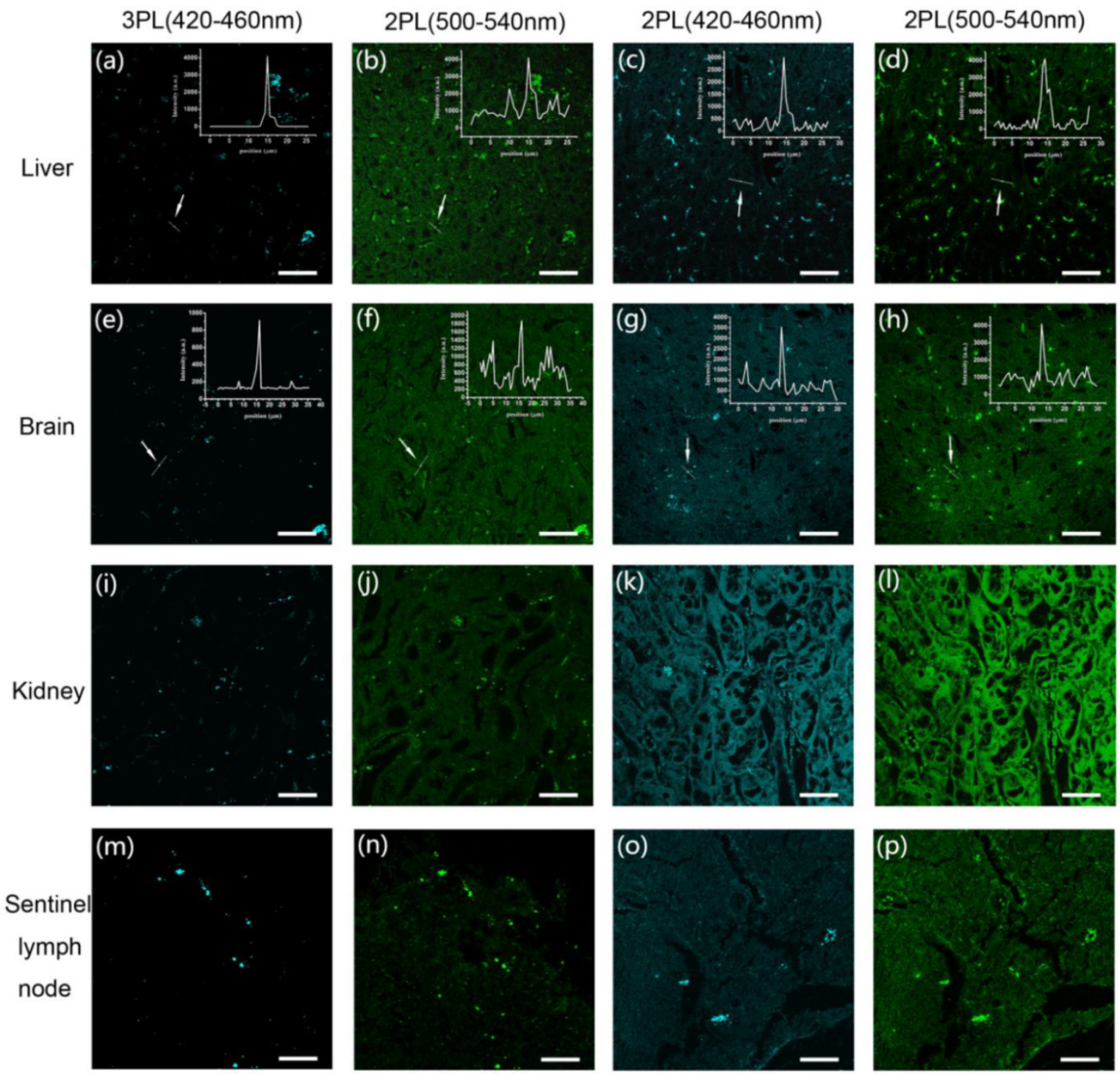

Mouse treated with 1000GNRs
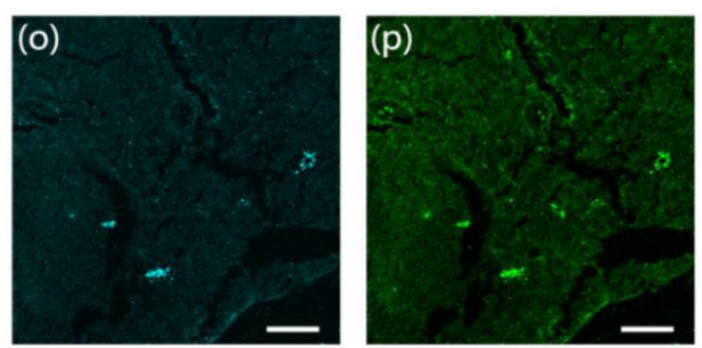

Mouse treated with 760GNRs

Figure 4. 3PL and 2PL images of tissue slices harvested from mice injected with PEG-1000GNRs and PEG-760GNRs. MPL from GNRs and autofluorescence from tissues were simultaneously acquired. The bright spots indicate the presence of GNRs. Scale bars: $50 \mu \mathrm{m}$.

PEG-760GNRs and PEG-1000GNRs were intravenously injected into normal mice and tumor-bearing mice via the tail vein, respectively, and the two GNR samples were also intradermally injected into the left forepaw pad of the mice. All the control mice (normal and tumor-bearing mice) were treated with equal amount of saline (see Materials and Methods for details). For multi-photon excitation of PEG-1000GNRs and PEG-760GNRs in the tissues, $1000 \mathrm{~nm}$ and $760 \mathrm{~nm}$ fs laser beams were focused onto the sample by a $20 \times$ objective $(\mathrm{NA}=0.75)$, respectively. The MPL from GNRs and autofluorescence from tissues were simultaneously acquired by the same objective. Representative 3PL and 2PL images of liver, brain, kidney and sentinel lymph node (SLN) were shown in Figure 4, in which the GNRs appeared as bright dots in the sliced tissues. Both
PEG-1000GNRs and PEG-760GNRs showed high accumulation in the mouse liver and kidney, which are part of the reticuloendothelial system (RES) responsible for the clearance of foreign materials by macrophage uptake [28]. In the brain tissues, GNRs were observed to be situated in and around the blood vessels, suggesting that they had been transported to the brain via blood circulation. GNRs also accumulated much in the SLN as a result of the lymph vessels transportation, and the mapping of the SLN is a key process in SLN biopsy for cancer staging and surgery $[29,30]$. Due to the fast processing of RES and blood circulation, the major organs were collected $0.5 \mathrm{~h}$ after the GNRs administration. As shown in the left two columns in Figure 4, under $1000 \mathrm{~nm}$ fs laser excitation, the autofluorescence from tissues was clearly observed in the $500-540 \mathrm{~nm}$ channel (corresponding to 
the 2PL channel) and hardly observed in the 420-460 $\mathrm{nm}$ channel (corresponding to the 3PL channel), whereas the 3PL (420-460 nm) from GNRs can be visualized with very high contrast. In addition, the line profiles of signal intensity in Figure 4a and 4e (insets) showed a relatively higher signal-to-background ratio of 3PL than that of 2PL (according to the line profiles in insets of Figure $4 \mathrm{~b}$ and 4f). Under $760 \mathrm{~nm}$ fs laser excitation, 2PL images in the right two columns all exhibited obvious autofluorescence, reducing the contrast of 760GNRs distributed in tissues. One thing worth noting is that the excitation power of the $760 \mathrm{~nm}$ fs laser focused on the sample is only $2 \mathrm{~mW}$. However, very strong autofluorescence from tissues was still excited. As for the control, no bright dots of GNRs were observed in images of the tissues explanted from mice injected with saline (Additional file 1: Figure S5).

For tumor-bearing mice, as shown in Figure 5 (top two rows), the bright MPL signals in tumor tissues indicated that substantial GNRs aggregated in the tumor. It illustrated that PEG-GNRs showed a high propensity to accumulate in the tumor via enhanced permeability and retention (EPR) effects. It is a time-dependent process and an appropriate long time $(48 \mathrm{~h})$ interaction is necessary for GNRs accumulation in tumors $[44,45]$. The black clusters in the bright field images confirmed that the PEG-GNRs accumulated into large aggregates within the tumor tissue post-injection and the white dotted lines indicated the boundaries of the tumor tissues (Figure 5a, 5e). The inset of Figure 5a is a magnification of the white box area and shows a clear boundary of tumor tissue (large and clear bright field images of tumor tissues are demonstrated in Additional file 1: Figure S6 and S7). Similarly, the accumulated 1000GNRs exhibited bright 3PL under $1000 \mathrm{~nm}$ fs laser excitation, and the autofluorescence from tissue is very weak (Figure $5 b$ ). However, autofluorescence (500-540 nm) of tumor tissue under $1000 \mathrm{~nm}$ fs excitation and autofluorescence (420-460 nm and 500-540 nm) of tumor tissue under $760 \mathrm{~nm}$ fs excitation, were very distinct (Figure $5 c, 5 f, 5 g)$, which can be further confirmed by the line profiles of MPL signal intensity (inset of Figure $5 c, 5 f$, $5 \mathrm{~g}$ ). The 3PL images provided evidence that GNRs had undergone an EPR directed aggregation process in the tumor tissue. The GNRs passively diffused into the tumor and were trapped within the extracellular space within the tissue. For the control, no bright dots were observed in images of the tumor tissues explanted from mice injected with saline. Histological images of tumor tissues harvested from the same area of tumor samples for imaging (tissue collected $48 \mathrm{~h}$ after GNRs injection) confirmed that there were no abnormalities or lesions of the tumor during the targeting of GNRs.

Although the accumulation of GNRs in tissues could be detected by TEM images, MPL imaging is an easier and quicker method. The high contrast of 3PL imaging is attributed to the low autofluorescence under $1000 \mathrm{~nm}$ fs excitation. First, the autofluorescence in the 420-460 $\mathrm{nm}$ channels is usually weaker than that in 500-540 $\mathrm{nm}$ channels, resulting in intrinsic lower background. However, we also notice that the intensity of two-photon autofluorescence (420-460 $\mathrm{nm}$ ) under $760 \mathrm{~nm}$ fs excitation is indeed stronger than three-photon autofluorescence (420-460 nm) under $1000 \mathrm{~nm}$ fs excitation [46]. Thus, the lower autofluorescence background of $1000 \mathrm{~nm}$ excited 3PL imaging is attributed to shorter emission wavelength, as well as higher order of nonlinear optical effect. These indicate that $1000 \mathrm{~nm}$ fs excitation is advantageous for imaging with low autofluorescence. Although the 3PL of 1000GNRs is less than the 2PL of 1000GNRs under $1000 \mathrm{~nm}$ fs excitation, as well as the 2PL of 760GNRs under $760 \mathrm{~nm}$ fs excitation, the weaker autofluorescence can make 1000GNR-assisted 3PL microscopy under $1000 \mathrm{~nm}$ fs excitation have a higher signal to background ratio in tissue imaging, compared to 1000GNR-assisted 2PL microscopy under $1000 \mathrm{~nm}$ fs excitation, as well as 760GNR-assisted 2PL microscopy under $760 \mathrm{~nm}$ fs excitation. 3PL imaging of GNRs holds great potential in nanoparticle-assisted tumor diagnostic and therapeutic applications [39-44, 47-49].

\section{In vivo 3PL imaging of GNRs in mouse ear blood vessels}

First, we used a mouse ear vessel model to examine the capability of GNRs in in vivo 3PL imaging (see Materials and Methods for details). Figure 6a-f showed 3PL images of 1000GNRs at various depths of mouse ear skin, under the fs excitation of $1000 \mathrm{~nm}$. Aside from the small capillaries located throughout the dermis, major veins and arteries located deeper within the dermis could also be observed. Figure $6 \mathrm{~h}$ showed a 3D reconstructed image of the blood vasculature network within a region of the ear dermis. In the control group, no obvious autofluorescence from ear vessels could be detected even when the excitation power was very high (Additional file 1: Figure S8). Combing the results from both control and experiment groups, we confirm that the MPL signals detected from the ear blood vessels of mice, which were treated with GNRs, were indeed from the GNRs flowing in the blood vessels. GNRs hold great promise to serve as an alternative contrast agent for intravital vasculature imaging. 


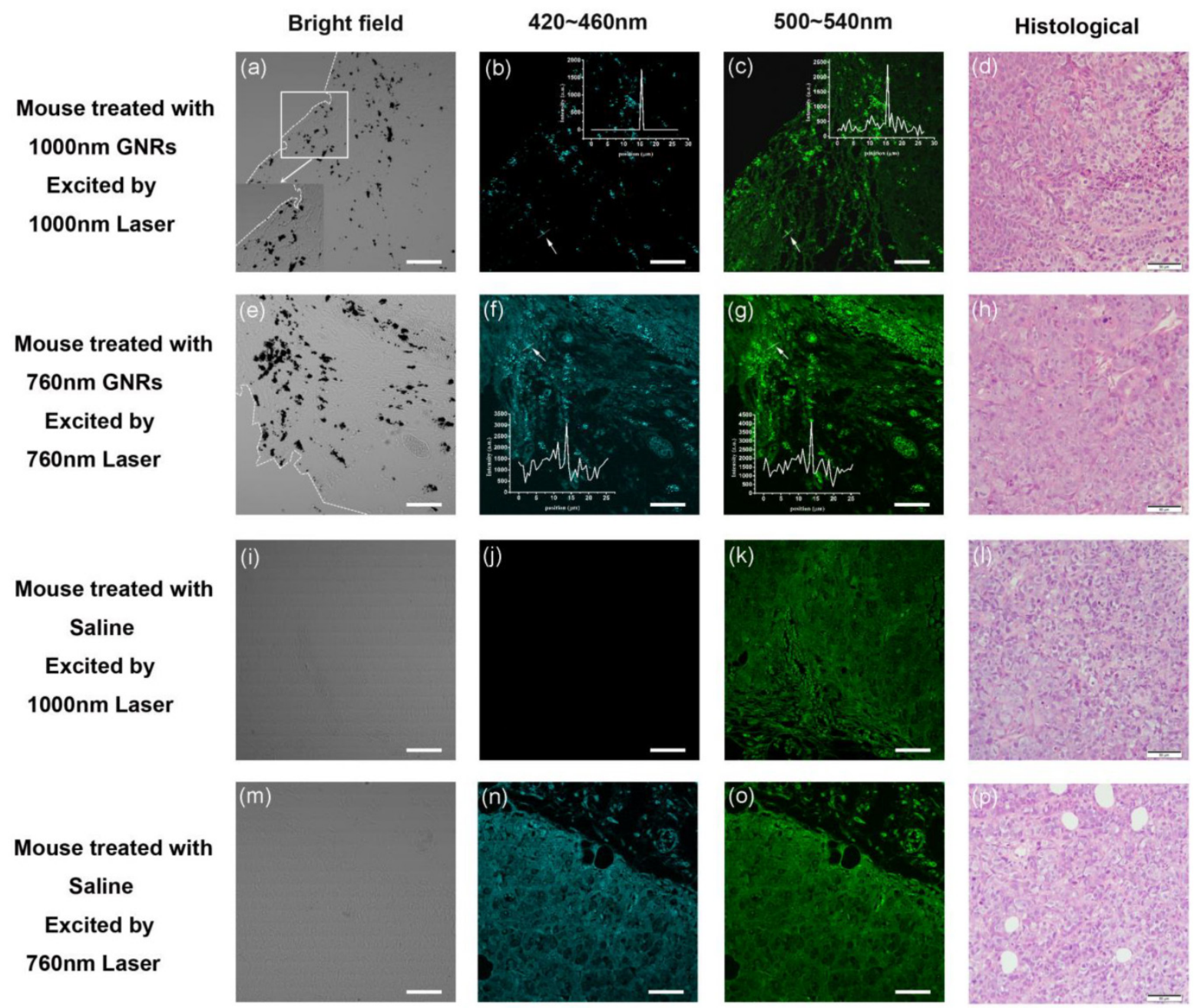

Figure 5. 3PL and 2PL images of tumor tissue slices harvested from mice injected with PEG-1000GNRs, PEG-760GNRs and saline. The white dotted lines indicate the boundaries of the tumor tissues. Scale bars: $50 \mu \mathrm{m}$.

\section{Simulation of the intensity distribution of focal spots of $1000 \mathrm{~nm}$ and $760 \mathrm{~nm}$ in biological tis- sue}

Laser scanning microscopy (LSM), such as confocal microscopes and multi-photon microscopes, is the most common setup for imaging biological tissue [50]. To estimate the ability of LSM for deep in vivo imaging, vectorial electric field Monte Carlo (EMC) simulation, which can simulate the scattering and absorption of the electric field in biological tissue, was utilized to simulate the optical focal spots of $1000 \mathrm{~nm}$ and $760 \mathrm{~nm}$ at different depths of biological tissue [51]. The tissue simulated here was assumed to be an aqueous phantom containing $1-\mu \mathrm{m}$-diameter scattering beads at a concentration of 0.1044 spheres/micron [52]. $760 \mathrm{~nm}$ and $1000 \mathrm{~nm}$ laser wavelengths were selected for simulation as they were used in our nonlinear optical imaging experiment. Figure 7 shows the intensity distribution of focal spots at various depths. Initially, the size of the focal spot of the $1000 \mathrm{~nm}$ beam is larger than that of the $760 \mathrm{~nm}$ beam due to the diffraction effect. However, the scattering coefficient of the $760 \mathrm{~nm}$ light is larger than that of $1000 \mathrm{~nm}$ light. As the focal depth gets larger, the number of scattering events for the propagation of the $760 \mathrm{~nm}$ light increases rapidly, deteriorating the focal intensity in the biological tissues [53]. As shown in Figure 7, the intensity of the focal spot of $1000 \mathrm{~nm}$ light is always stronger than that of $760 \mathrm{~nm}$ light from depths of 100 $\mu \mathrm{m}$ to $700 \mu \mathrm{m}$, suggesting lower attenuation for longer-wavelength light in tissues. In particular, the intensity ratio of $1000 \mathrm{~nm}$ to $760 \mathrm{~nm}$ at a depth of 400 $\mu \mathrm{m}$ was around 6.9 and increased to about 26 at a depth of $700 \mu \mathrm{m}$ (Figure 7h), indicating that the 1000 $\mathrm{nm}$ fs laser was more suitable than the $760 \mathrm{~nm}$ fs laser for optical excitation in deep multi-photon imaging. 

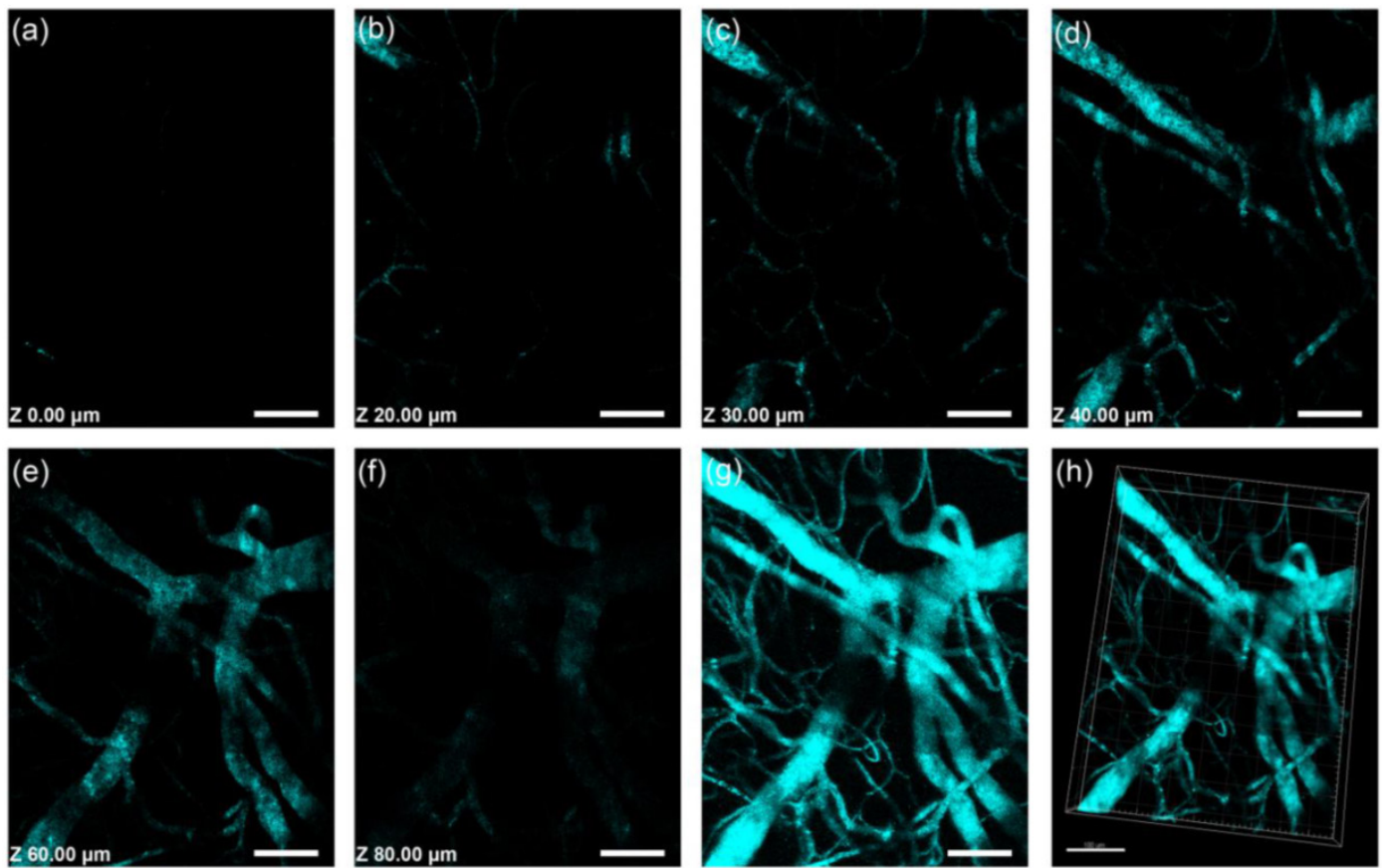

Figure 6. Intravital 3PL images of PEG-1000GNR-stained mouse ear blood vessels. (a)-(f) Images at various vertical depths of mouse ear skin. (g) Stacked 3PL image from a depth of $0 \mu \mathrm{m}$ to $80 \mu \mathrm{m}$. (h) $3 \mathrm{D}$ reconstructed image showing blood vessels in mouse ear skin. $\lambda_{\mathrm{ex}}=1000 \mathrm{~nm}$. Signal collected within $420-460 \mathrm{~nm}$. Scale bars: $100 \mu \mathrm{m}$.
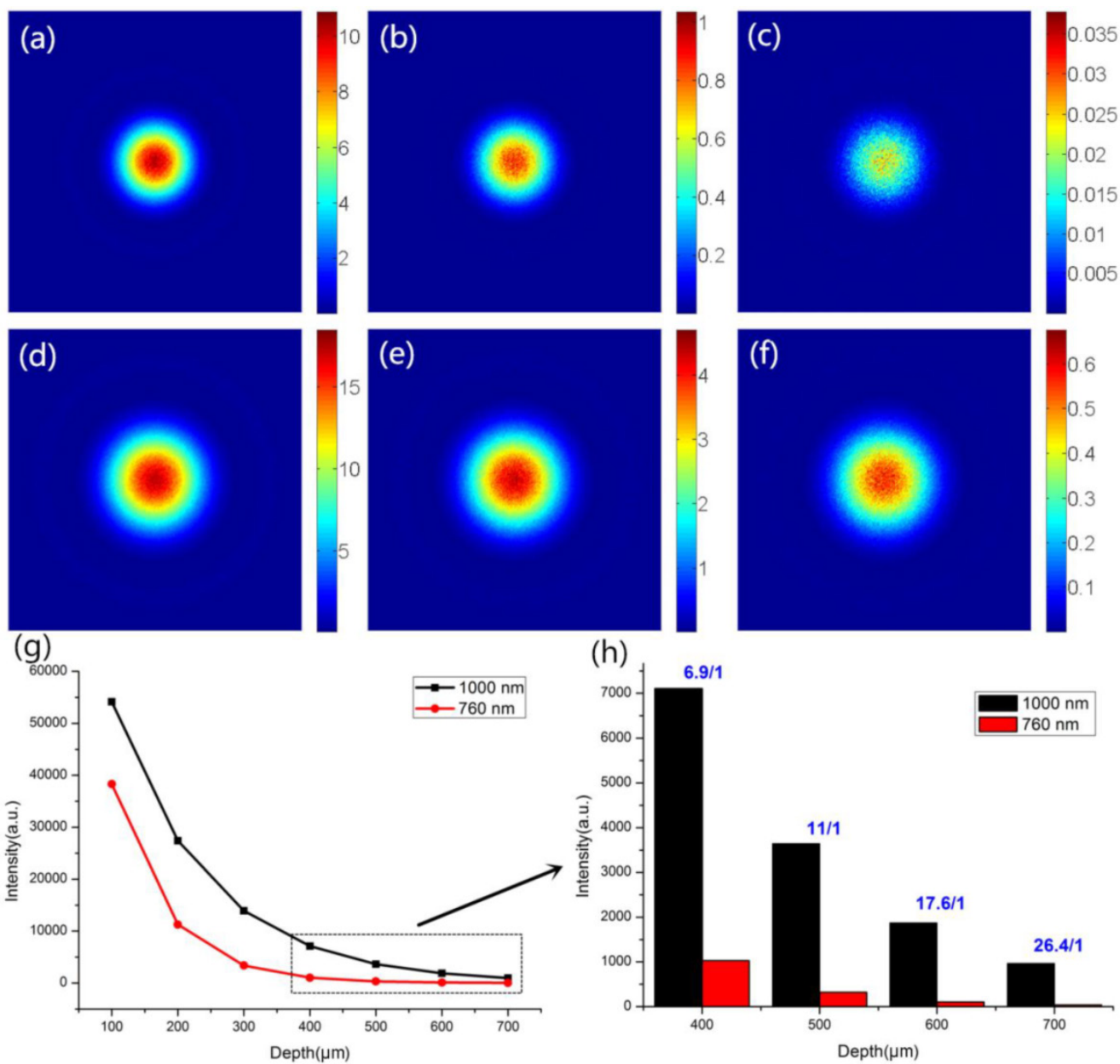

Figure 7. Simulation of the intensity distribution of $760 \mathrm{~nm}$ and $1000 \mathrm{~nm}$ light in biological tissue. The simulated size of the focal spots of (a-c) $760 \mathrm{~nm}$ and (d-f) 1000 $\mathrm{nm}$ light at depths of $100 \mu \mathrm{m}, 300 \mu \mathrm{m}$ and $600 \mu \mathrm{m}$. (g) The simulated intensity distribution trend of focal spots at various depths of biological tissue. (h) The light intensity ratio of $1000 \mathrm{~nm}$ to $760 \mathrm{~nm}$ at depths of $400 \mu \mathrm{m}, 500 \mu \mathrm{m}, 600 \mu \mathrm{m}$ and $700 \mu \mathrm{m}$. 


\section{PL and 2PL imaging of GNRs in mouse brain blood vessels}

In vivo imaging of mouse brain vessels was performed to demonstrate the capability of deeper tissue imaging in the "optical tissue window" by using 3PL of GNRs. The BALB/c mice ( 5 weeks old, male) were used for all the experiments. MPL signal intensity is higher when fs excitation wavelength is closer to the longitudinal LSPR of GNRs, given that the fs excitation power is set to be the same. Thus, two types of PEG-modified GNRs (PEG-1000GNRs and PEG-760GNRs) were used and compared for deep tissue MPL imaging, with excitation wavelengths at $1000 \mathrm{~nm}$ and $760 \mathrm{~nm}$ respectively.

We first performed a cranial window surgery on the mouse brain. To ensure an accurate comparison, $200 \mu \mathrm{l}$ of a $1 \times$ PBS mixed solution of PEG-760GNRs $(100 \mu \mathrm{l}, 25 \mathrm{nM})$ and PEG-1000GNRs $(100 \mu \mathrm{l}, 25 \mathrm{nM})$ were intravenously injected into the male BALB/c mice (see Materials and Methods for details). The absorption spectrum of the mixed PEG-GNRs showed two distinct LSPR peaks at $760 \mathrm{~nm}$ and $1000 \mathrm{~nm}$, which were used to couple the fs excitation wavelengths of $760 \mathrm{~nm}$ and $1000 \mathrm{~nm}$, respectively (Additional file 1: Figure S9). MPL process of the GNRs is plasmon-enhanced, and the brightness of the MPL reaches maximum when excited at the LSPR peak [16], thus bright MPL of GNRs can be excited by a fs laser at a low power when its wavelength is coupled with the longitudinal LSPR band of the GNRs. However, higher power is required to excite MPL of the GNRs when fs excitation wavelength is far away from the absorbance band of the GNRs. The MPL of 1000GNRs excited by $1000 \mathrm{~nm}$ fs laser and 760GNRs excited by $760 \mathrm{~nm}$ fs laser could be clearly observed at a low power $(5 \mathrm{~mW})$ in the imaging of intravital mice's ear blood vessels (Additional file 1: Figure S10). However, the MPL of $1000 \mathrm{GNRs}$ excited by $760 \mathrm{~nm}$ fs laser and 760GNRs excited by $1000 \mathrm{~nm}$ fs laser was not efficient even at very high excitation powers (25 $\mathrm{mW})$. Therefore, MPL of the GNRs excited with fs excitation wavelength far away from their LSPR bands are very weak, and the MPL of a mixture of two kinds of GNRs excited by fs laser with two different wavelengths did not have any influence on each other. The surgery treated area of the brain was chosen for multi-photon imaging and comparison. For two-photon excitation of PEG-760GNRs and three-photon excitation of PEG-1000GNRs, $760 \mathrm{~nm}$ and $1000 \mathrm{~nm}$ fs lasers were adopted, respectively, and two filters within the 500-540 nm and 575-630 nm were used to extract the $2 \mathrm{PL}$ emission signals and a filter within 420-460 nm was used to collect 3PL emission signals from GNRs. The average powers required for MPL imaging at the surface of the brain was $2 \mathrm{~mW}$ for $760 \mathrm{~nm}$ excitation and $3 \mathrm{~mW}$ for 1000 $\mathrm{nm}$ excitation, and the powers were gradually increased when the imaging depths were increased, which can ensure that similar signal intensity and signal-to-noise ratio were achieved at different imaging depths.

We acquired a $429-\mu \mathrm{m}$-deep stack for 2PL of

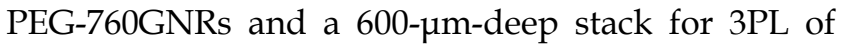
PEG-1000GNRs, respectively, taken with a 1.5- $\mu \mathrm{m}$-step depth. Figure $8 \mathrm{j}$ and $9 \mathrm{j}$ showed three-dimensional reconstructive MPL images of the mice brain vasculature imaged through a cranial window (for 3D reconstructed vessels network with $360^{\circ}$ rotation, see Video 1 in Additional file 2). Z-sections ( $\mathrm{x}-\mathrm{y}$ frames) at various depths (see Video 2 in Additional file 3) showed that high contrast imaging of the major blood vessels and smaller capillaries in the pia mater could be visualized clearly with the help of 3PL of the GNRs. The maximum imaging depth of the PEG-1000GNRs with fs excitation at 1000 $\mathrm{nm}$ reached $600 \mu \mathrm{m}$ below the brain surface, approximately $170 \mu \mathrm{m}$ deeper than that of PEG-760GNRs with fs excitation at $760 \mathrm{~nm}$. Microvasculature deep in the brain, beyond the pia matter $(>400 \mu \mathrm{m})$, could still be visualized with high resolution and contrast for the PEG-1000GNRs. At a depth of $600 \mu \mathrm{m}$, the small capillaries could still be detected with good contrast with the full power $(\sim 60 \mathrm{~mW}$ at the surface of the sample) of the excitation wavelength (Figure 9h). Comparing Figure 8j with Figure 9j, we could visualize more small capillaries in the three-dimensional reconstruction of 3PL of the PEG-1000GNRs with good resolution, while for PEG-760GNRs, these small capillaries could not be excited or detected (although they do exist). The reason why 3PL imaging of PEG-1000GNRs under 1000 $\mathrm{nm}$ fs excitation has a larger imaging depth can be found in our simulation results in Figure 7. Overall, considering the water absorption and tissue scattering, $1000 \mathrm{~nm}$ light has a better focusing effect than 760 $\mathrm{nm}$ light deep in the biological sample (e.g. the intensity ratio of $1000 \mathrm{~nm}$ to $760 \mathrm{~nm}$ at a depth of $600 \mu \mathrm{m}$ was around 17.6). As MPL capability is closely related to the power density at the focal spot, 3PL of PEG-1000GNRs under $1000 \mathrm{~nm}$ fs excitation can have a higher efficiency than 2PL of PEG-760GNRs under $760 \mathrm{~nm}$. In the control group, no obvious autofluorescence from brain vessels could be detected even when the excitation power was very high (Additional file 1: Figure S11).

During the 3PL imaging of PEG-1000GNRs, 2PL signals from PEG-1000GNRs excited by $1000 \mathrm{~nm}$ (within the 500-540 $\mathrm{nm}$ and 575-630 nm ranges) were also collected. The maximum 2PL imaging depth of 
the PEG-1000GNRs reached $700 \mu \mathrm{m}, 100 \mu \mathrm{m}$ deeper than that of 3PL imaging of PEG-1000GNRs and 270 $\mu \mathrm{m}$ deeper than that of 2PL imaging of PEG-760GNRs excited by $760 \mathrm{~nm}$ (Additional file 1: Figure S12). However, under the fs excitation of the same wavelength, 3PL imaging has a higher spatial resolution. In our case, the spatial resolution of 3PL imaging of PEG-1000GNRs (excited by the $1000 \mathrm{fs}$ laser) is $288 \mathrm{~nm}$ $(\lambda /(2 \sqrt{3} \mathrm{NA}, 288 \mathrm{~nm}$ for $\lambda=1000 \mathrm{~nm}, \mathrm{NA}=1)$, which is higher than that of 2PL imaging of PEG-1000GNRs $(\lambda /(2 \sqrt{2} \mathrm{NA}, 353 \mathrm{~nm}$ for $\lambda=1000 \mathrm{~nm}, \mathrm{NA}=1$, also excited by the $1000 \mathrm{fs}$ laser) [54, 55]. In addition, owing to the significant improvement in the overall excitation localization, three-photon excitation can reduce the out-of-focus background and improving the signal-to-noise ratio by orders of magnitude when compared to two-photon excitation [24]. These results demonstrated that 3PL of GNRs excited by a long wavelength fs laser is advantageous for high contrast and deep in vivo imaging.
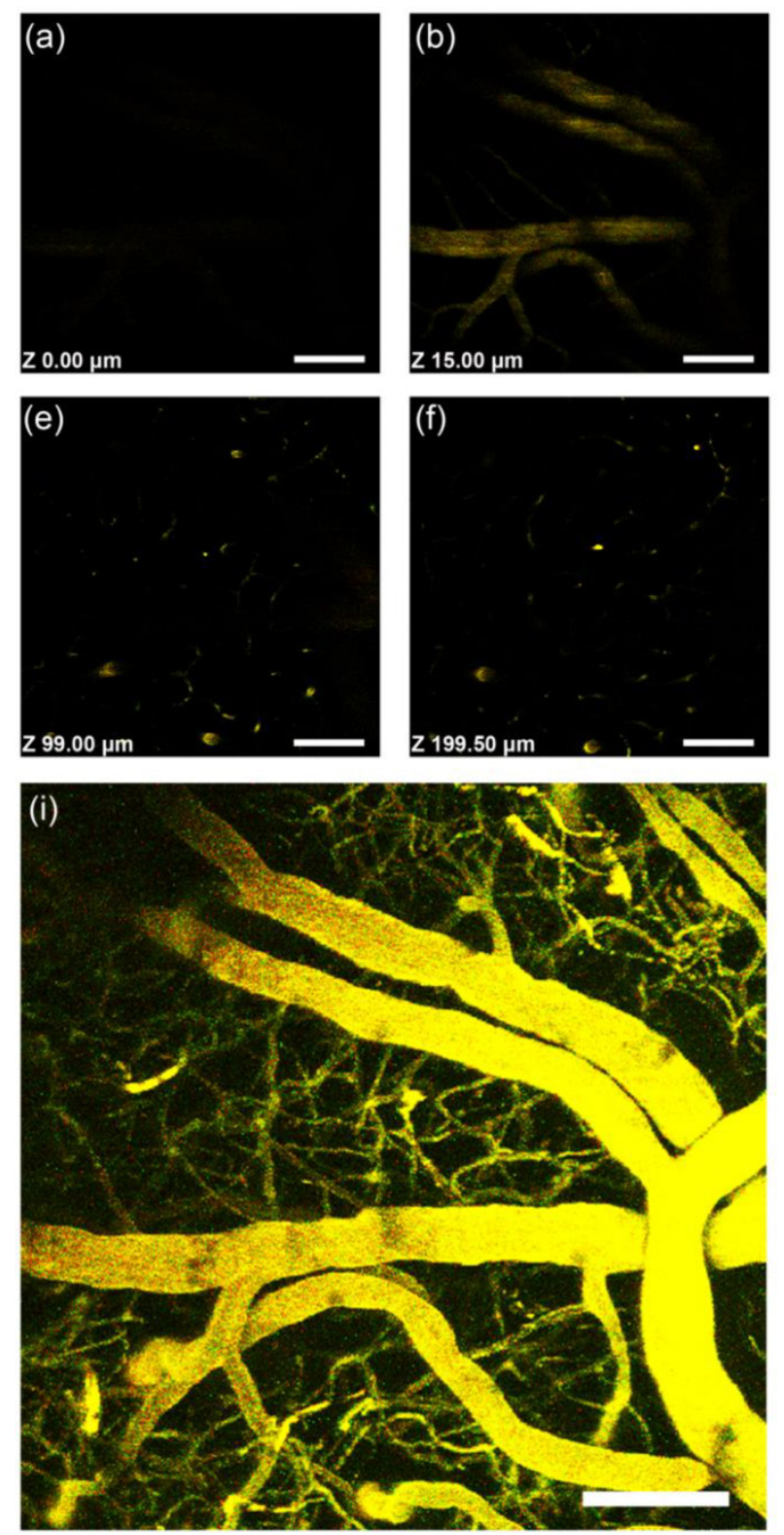

Figure 8. Intravital 2PL images of PEG-760GNR-stained mouse brain blood vessels. (a)-(h) Images at various vertical depths of the mouse brain. (Inset, h) The line profile of the 2PL signal intensity from the point indicated by the white arrow illustrated that the 2PL signals could be detected with high contrast at a depth of 429 $\mu \mathrm{m}$. (i) Stacked $2 \mathrm{PL}$ images from a depth of $0 \mu \mathrm{m}$ to $429 \mu \mathrm{m}$. (j) $3 \mathrm{D}$ reconstructed image showing blood vessels in the mouse brain. $\lambda_{\text {ex }}=760 \mathrm{~nm}$. Signal collected within 500-540 nm and 575-630 nm. Scale bars: $100 \mu \mathrm{m}$.
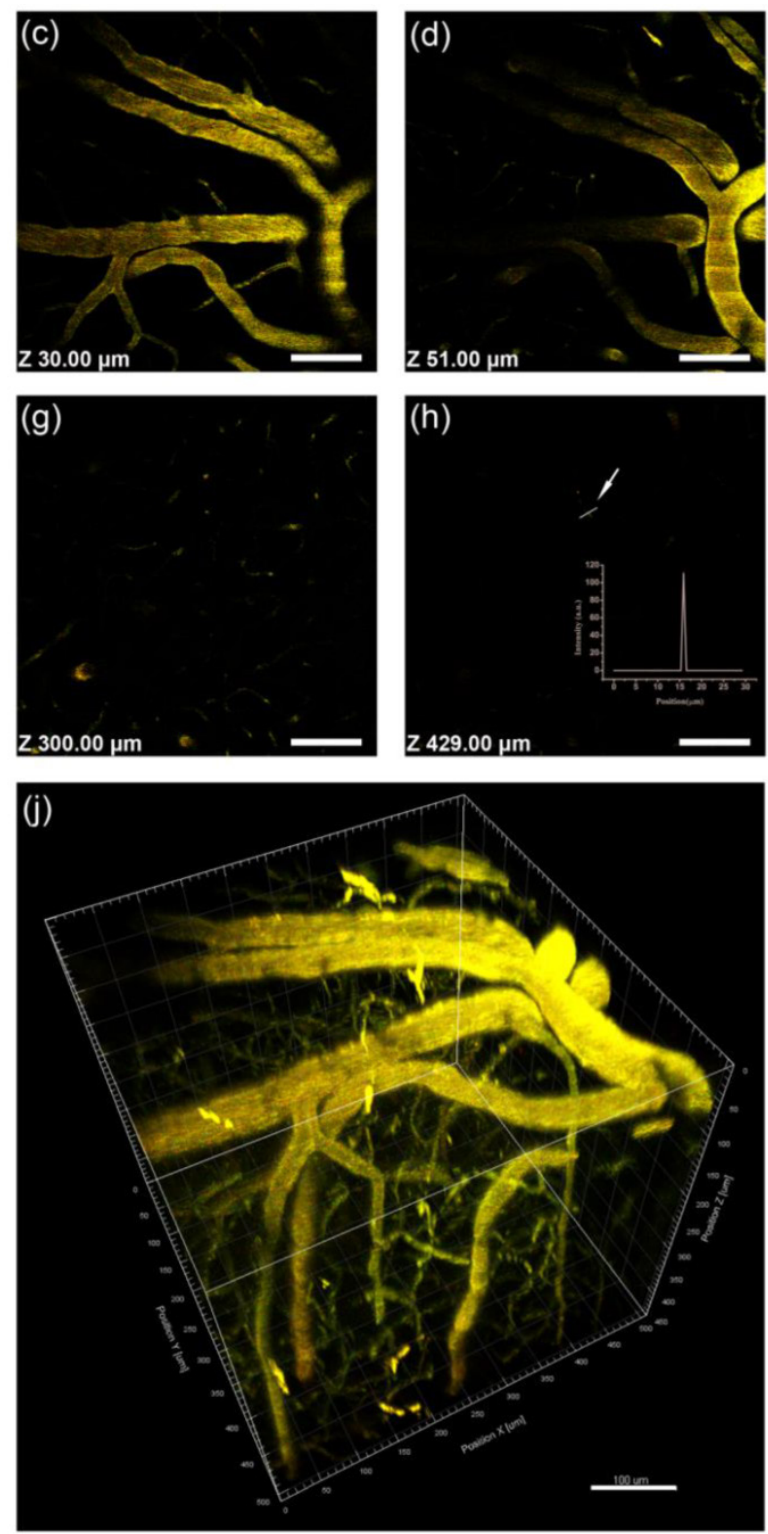

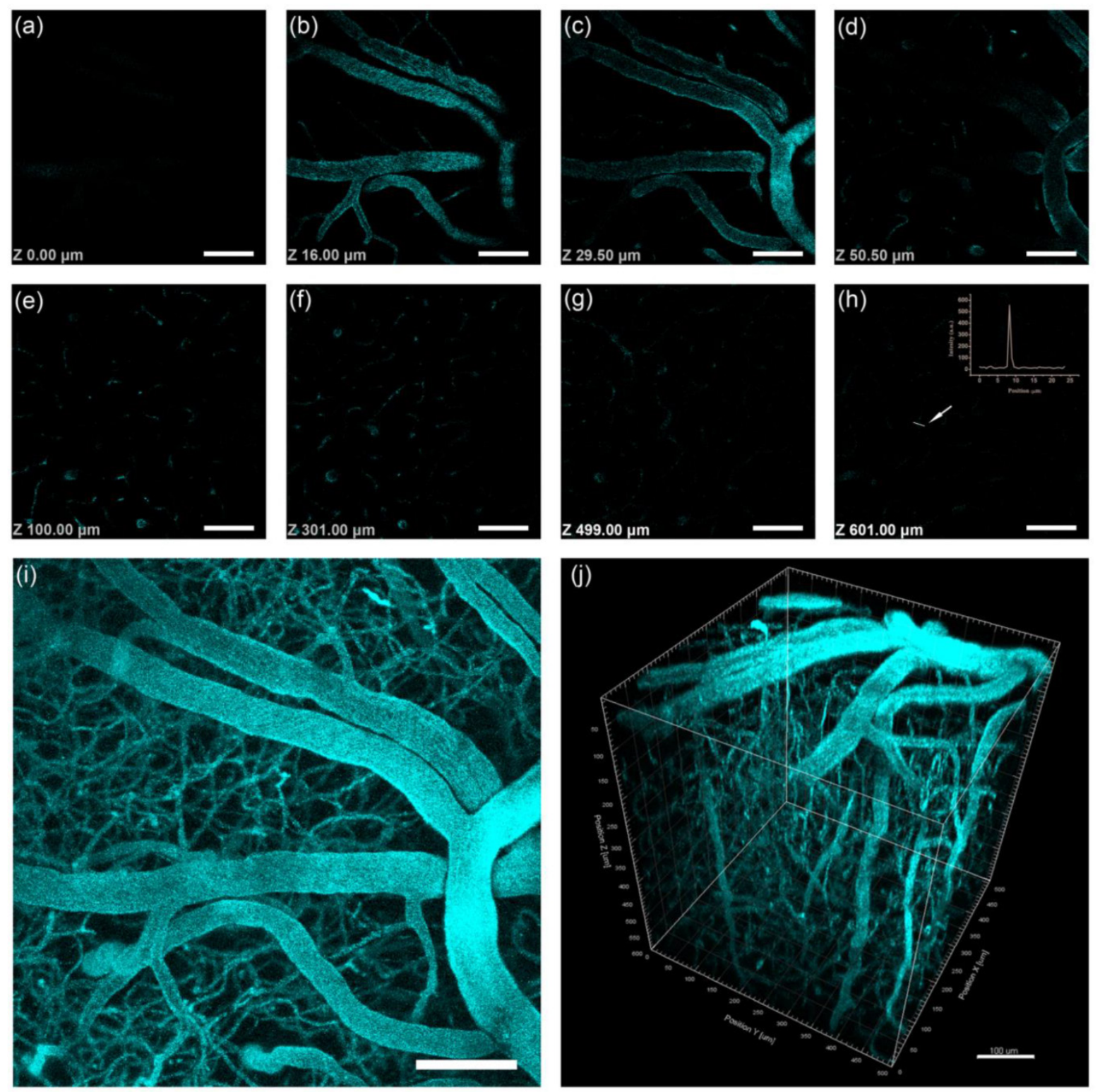

Figure 9. Intravital 3PL images of PEG-1000GNR-stained mouse brain blood vessels. (a)-(h) Images at various vertical depths of the mouse brain. (Inset, h) The line profile of the 3PL signal intensity from the point indicated by the white arrow illustrated that the 3PL signals could be detected with high contrast at a depth of 601 $\mu \mathrm{m}$. (i) Stacked 3PL images from a depth of $0 \mu \mathrm{m}$ to $601 \mu \mathrm{m}$. (j) 3D reconstructed image showing blood vessels in the mouse brain. $\lambda_{\text {ex }}=1000 \mathrm{~nm}$. Signal collected within $420-460 \mathrm{~nm}$. Scale bars: $100 \mu \mathrm{m}$.

\section{Conclusions}

In summary, gold nanorods with low and high aspect ratios were synthesized by using two different protocols based on seed-mediated methods in aqueous solution, and their two-photon and three-photon luminescence were clearly observed and compared in in vitro and in vivo imaging. PEG modified CTAB/NaOL-GNRs exhibited high chemical stability under various $\mathrm{pH}$ values and negligible toxicity. In in vitro tissue imaging, three-photon luminescence imaging clearly illustrated the distribution of PEG-GNRs in major organs and tumor tissue with high contrast. Vectorial electric field Monte Carlo (EMC) was uti- lized to simulate the focal spots in the biological tissue, and the results confirmed that the longer wavelength laser was a more suitable optical source in deep multi-photon imaging. By tuning the longitudinal plasmonic bands of GNRs to absorb long-wavelength fs excitation in the optical tissue window, 3PL imaging of PEG-GNRs in in vivo imaging of mouse ear and brain vessels was achieved with a maximum depth of $600 \mu \mathrm{m}$. The main advantages of developing such wavelength-selective plasmon-enhanced MPL probes for biological applications are as follows: (i) the tunability of LSPR bands by controlling the aspect ratio of GNRs makes it easy for selecting an appropriate NIR wavelength to achieve deep in vivo imaging; (ii) PEG 
modified GNRs are more biocompatible for in vivo applications than some toxic nanomaterials; (iii) excited by a longer wavelength laser, which penetrates deep into tissue, 3PL imaging of GNRs holds great potential in nanoparticle-assisted tumor diagnostic and therapeutic applications in deep tissues.

\section{Abbreviations}

GNRs: gold nanorods; fs: femtosecond; MPL: multi-photon luminescence; 3PL: three-photon luminescence; 2PL: two-photon luminescence; PEG: polyethylene glycol; SPR: surface plasmon resonance; NIR: near-infrared; EPR: enhanced permeability and retention; TEM: transmission electron microscopy; H\&E: hematoxylin- eosin.

\section{Supplementary Material}

Additional File 1:

Table S1 and Figures S1-S12.

http://www.thno.org/v05p0251s1.pdf

Additional File 2:

Video 1. http:/ / www.thno.org/v05p0251s2.avi

Additional File 3:

Video 2. http:/ / www.thno.org/v05p0251s3.avi

\section{Acknowledgements}

This work was supported by National Basic Research Program of China (973 Program; 2013CB834704 and 2011CB503700), the National Natural Science Foundation of China (61275190, 61008052, 91233208 and 61107017), the Program of Zhejiang Leading Team of Science and Technology Innovation (2010R50007) and the Fundamental Research Funds for the Central Universities. Shaowei Wang is grateful to Ms. S. S. Liu for help with multi-photon laser scanning confocal microscopy and $\mathrm{Mr}$. K. Wu for help with animal experiments.

\section{Competing Interests}

The authors have declared that no competing interest exists.

\section{References}

1. Hong G, Lee J C, Robinson J T, et al. Multifunctional in vivo vascular imaging using near-infrared II fluorescence. Nat Med. 2012; 18: 1841-1846.

2. Lee J H, Huh Y M, Jun Y, et al. Artificially engineered magnetic nanoparticles for ultra-sensitive molecular imaging. Nat Med. 2007; 13: 95-99.

3. Li N, Zhao P, Astruc D. Anisotropic Gold Nanoparticles: Synthesis, Properties, Applications, and Toxicity. Angew Chem Int Ed. 2014; 53: 1756-1789.

4. Chen H, Shao L, Li Q, et al. Gold nanorods and their plasmonic properties. Chem Soc Rev. 2013; 42: 2679-2724.

5. Huang X, Neretina S, El-Sayed M A. Gold nanorods: from synthesis and properties to biological and biomedical applications. Adv Mater. 2009; 21: 4880-4910.

6. Zhang Z, Wang J, Chen C. Gold nanorods based platforms for light-mediated theranostics. Theranostics. 2013; 3: 223-238.

7. Zijlstra P, Paulo P M R, Orrit M. Optical detection of single non-absorbing molecules using the surface plasmon resonance of a gold nanorod. Nat Nanotechnol. 2012; 7: 379-382.

8. Mayer K M, Lee S, Liao H, et al. A label-free immunoassay based upon localized surface plasmon resonance of gold nanorods. ACS Nano. 2008; 2: 687-692.
9. Qian J, Jiang L, Cai F, et al. Fluorescence-surface enhanced Raman scattering co-functionalized gold nanorods as near-infrared probes for purely optical in vivo imaging. Biomaterials. 2011; 32: 1601-1610.

10. Zhang Y, Qian J, Wang D, et al. Multifunctional gold nanorods with ultrahigh stability and tunability for in vivo fluorescence imaging, SERS detection, and photodynamic therapy. Angew Chem Int Ed. 2013; 52: 1148-1151.

11. Jang B, Park J Y, Tung C H, et al. Gold nanorod-photosensitizer complex for near-infrared fluorescence imaging and photodynamic/photothermal therapy in vivo. ACS Nano. 2011; 5: 1086-1094

12. Huang X, El-Sayed I H, Qian W, et al. Cancer cell imaging and photothermal therapy in the near-infrared region by using gold nanorods. J Am Chem Soc. 2006; 128: 2115-2120.

13. Oldenburg A L, Hansen M N, Zweifel D A, et al. Plasmon-resonant gold nanorods as low backscattering albedo contrast agents for optical coherence tomography. Opt Express. 2006; 14: 6724-6738.

14. Eghtedari M, Oraevsky A, Copland J A, et al. High sensitivity of in vivo detection of gold nanorods using a laser optoacoustic imaging system. Nano Lett. 2007; 7: 1914-1918.

15. Vonnemann J, Beziere N, Böttcher C, et al. Polyglycerolsulfate Functionalized Gold Nanorods as Optoacoustic Signal Nanoamplifiers for In Vivo Bioimaging of Rheumatoid Arthritis. Theranostics. 2014; 4:629-641.

16. Wang H, Huff T B, Zweifel D A, et al. In vitro and in vivo two-photon luminescence imaging of single gold nanorods. Proc Natl Acad Sci U.S.A. 2005; 102: 15752-15756.

17. Durr N J, Larson T, Smith D K, et al. Two-photon luminescence imaging of cancer cells using molecularly targeted gold nanorods. Nano Lett. 2007; 7: 941-945.

18. Li J L, Gu M. Surface plasmonic gold nanorods for enhanced two-photon microscopic imaging and apoptosis induction of cancer cells. Biomaterials. 2010; 31: 9492-9498.

19. Helmchen F, Denk W. Deep tissue two-photon microscopy. Nat Methods. 2005; 2: 932-940

20. Tozer G M, Ameer-Beg S M, Baker J, et al. Intravital imaging of tumour vascular networks using multi-photon fluorescence microscopy. Adv Drug Deliver Rev. 2005; 57: 135-152

21. Hoover E E, Squier J A. Advances in multiphoton microscopy technology. Nat Photonics. 2013; 7: 93-101.

22. $\mathrm{Xu} \mathrm{C}$, Wise F W. Recent advances in fibre lasers for nonlinear microscopy. Nat Photonics. 2013; 7: 875-882.

23. Kobat D, Durst M E, Nishimura N, et al. Deep tissue multiphoton microscopy using longer wavelength excitation. Opt Express. 2009; 17: 13354-13364.

24. Horton N G, Wang K, Kobat D, et al. In vivo three-photon microscopy of subcortical structures within an intact mouse brain. Nat Photonics. 2013; 7: 205-209.

25. Hu K W, Liu T M, Chung K Y, et al. Efficient near-IR hyperthermia and intense nonlinear optical imaging contrast on the gold nanorod-in-shell nanostructures. J Am Chem Soc. 2009; 131: 14186-14187.

26. Nikoobakht B, El-Sayed M A. Preparation and growth mechanism of gold nanorods (NRs) using seed-mediated growth method. Chem Mater. 2003; 15: 1957-1962.

27. Ye X, Zheng C, Chen J, et al. Using binary surfactant mixtures to simultaneously improve the dimensional tunability and monodispersity in the seeded growth of gold nanorods. Nano Lett. 2013; 13: 765-771.

28. Khlebtsov N, Dykman L. Biodistribution and toxicity of engineered gold nanoparticles: a review of in vitro and in vivo studies. Chem Soc Rev. 2011; 40: 1647-1671.

29. Kim S, Lim Y T, Soltesz E G, et al. Near-infrared fluorescent type II quantum dots for sentinel lymph node mapping. Nat Biotechnol. 2004; 22: 93-97.

30. Kim T, Giuliano AE, Lyman GH. Lymphatic mapping and sentinel lymph node biopsy in early-stage breast carcinoma - A metaanalysis. Cancer. 2006; 106: 4-16.

31. Huang G, Yang S, Yuan Y, Xing D. Combining x-ray and photoacoustics for in vivo tumor imaging with gold nanorods. Appl Phys Lett. 2011; 99: 123701.

32. Tsai M F, Chang S H G, Cheng F Y, et al. Au Nanorod Design as Light-Absorber in the First and Second Biological Near-Infrared Windows for in vivo Photothermal Therapy. ACS Nano. 2013; 7: 5330-5342.

33. Qiu Y, Liu Y, Wang L, et al. Surface chemistry and aspect ratio mediated cellular uptake of Au nanorods. Biomaterials. 2010; 31: 7606-7619.

34. Niidome T, Yamagata M, Okamoto $Y$, et al. PEG-modified gold nanorods with a stealth character for in vivo applications. J Control Release. 2006; 114: 343-347.

35. Imura $\mathrm{K}$, Okamoto $\mathrm{H}$. Properties of photoluminescence from single gold nanorods induced by near-field two-photon excitation. J Phys Chem C. 2009; 113: 11756-11759.

36. Imura $\mathrm{K}$, Nagahara T, Okamoto H. Near-field two-photon-induced photoluminescence from single gold nanorods and imaging of plasmon modes. J Phys Chem B. 2005; 109: 13214 -13220.

37. Wang D S, Hsu F Y, Lin C W. Surface plasmon effects on two photon luminescence of gold nanorods. Opt Express. 2009; 17: 11350-11359.

38. He G S, Markowicz P P, Lin T C, et al. Observation of stimulated emission by direct three-photon excitation. Nature. 2002; 415: 767-770.

39. Menon J U, Jadeja P, Tambe P, et al. Nanomaterials for photo-based diagnostic and therapeutic applications. Theranostics. 2013; 3: 152-166. 
40. Khlebtsov N, Bogatyrev V, Dykman L, et al. Analytical and theranostic applications of gold nanoparticles and multifunctional nanocomposites. Theranostics. 2013; 3: 167-180.

41. Huang H C, Barua S, Kay D B, et al. Simultaneous enhancement of photothermal stability and gene delivery efficacy of gold nanorods using polyelectrolytes. ACS Nano. 2009; 3: 2941-2952.

42. Alkilany A M, Thompson L B, Boulos S P, et al. Gold nanorods: their potential for photothermal therapeutics and drug delivery, tempered by the complexity of their biological interactions. Adv Drug Deliver Rev. 2012; 64:190-199.

43. Lukianova-Hleb E Y, Ren X, Sawant R R, et al. On-demand intracellular amplification of chemoradiation with cancer-specific plasmonic nanobubbles. Nature Med. 2014; 20: 778-784.

44. Agarwal A, Mackey M A, El-Sayed M A, Bellamkonda R V. Remote triggered release of Doxorubicin in tumors by synergistic application of thermosensitive liposomes and gold nanorods. ACS Nano. 2011; 5: 4919-4926.

45. Maeda H, Wu J, Sawa T, Matsumura Y, Hori K. Tumor vascular permeability and the EPR effect in macromolecular therapeutics: a review. J Controlled Release. 2000; 65: 271-284.

46. Zipfel W R, Williams R M, Christie R, et al. Live tissue intrinsic emission microscopy using multiphoton-excited native fluorescence and second harmonic generation. Proc Natl Acad Sci U.S.A. 2003; 100: 7075-7080.

47. Chen $\mathrm{H}$, Zhang $\mathrm{X}$, Dai S, et al. Multifunctional gold nanostar conjugates for tumor imaging and combined photothermal and chemo-therapy. Theranostics. 2013; 3: 633-649.

48. Zhang Z, Wang L, Wang J, et al. Mesoporous Silica-Coated Gold Nanorods as a Light-Mediated Multifunctional Theranostic Platform for Cancer Treatment. Adv Mater. 2012; 24: 1418-1423.

49. Zhang Z, Wang J, Nie X, et al. Near Infrared Laser Induced Targeted Cancer Therapy Using Thermo-Responsive Polymer Encapsulated Gold Nanorods. J Am Chem Soc. 2014; 136: 7317-7326.

50. Pawley J. Handbook of biological confocal microscopy Springer; 2010.

51. Hayakawa C K, Potma E O, Venugopalan V. Electric field Monte Carlo simulations of focal field distributions produced by tightly focused laser beams in tissues. Biomed Opt Express. 2011; 2: 278-299.

52. Cai F, Yu J, He S. Vectorial Electric Field Monte Caro Simulations for Focused Laser Beams $(800 \mathrm{Nm}-2220 \mathrm{Nm})$ in a Biological Sample. Prog Electromagn Res. 2013; 142: 667-681.

53. Hayakawa C K, Venugopalan V, Krishnamachari V V, et al. Amplitude and phase of tightly focused laser beams in turbid media. Phys Rev Lett. 2009; 103: 043903 .

54. Hell S W, Malak H M, Gryczynski I, et al. Three-photon excitation in fluorescence microscopy. J Biomed Opt. 1996; 1: 71-74.

55. Yu J H, Kwon S H, Petrášek Z, et al. High-resolution three-photon biomedical imaging using doped ZnS nanocrystals. Nat Mater. 2013; 12: 359-366. 